\title{
BIASED SWITCHING OF SMALL MAGNETIC PARTICLES
}

\author{
R.L. Stamps \\ Department of Physics, University of Western Australia, Nedlands, Western Australia, 6907 Australia \\ B. Hillebrands \\ Fachbereich Physik, Universität Kaiserslautern, 67653 Kaiserslautern, Germany
}

\begin{abstract}
High frequency switching of single domain, uniaxial magnetic particles is discussed in terms of transition rates controlled by a small transverse bias field. It is shown that fast switching times can be achieved using bias fields an order of magnitude smaller than the effective anisotropy field. Analytical expressions for the switching time are derived in special cases and general configurations of practical interest are examined using numerical simulations.
\end{abstract}

Modern techniques of thin film growth with nanometer precision geometrical control are capable of producing high quality arrays of submicron magnetic particles. ${ }^{1}$ This technology is of great interest for possible application in data storage and magnetic sensing devices, and presents several interesting challenges for understanding reversal processes. One important problem is the understanding and optimization of switching processes for time scales on the order of $100-1000 \mathrm{MHz}$ in soft materials. ${ }^{2}$

In this paper a new method for rapid, addressable switching using small magnetic fields is presented. The method is unusual in that the field strengths needed to trigger the switching can be nearly two orders of magnitude smaller than the effective magnetic anisotropy field of the material. The idea is to apply a small bias pulse field perpendicular to a larger switching field, thereby instigating reversal even for switching fields smaller than the effective anisotropy field of the material.

The geometry used in the following discussion is shown in Fig. 1. The switching of a single domain particle is examined, and only simultaneous coherent rotation of all the moments in a particle is considered. This approximation places upper bounds on the overall particle size appropriate for this theory, and temperatures are assumed low enough to ignore superparamagnetic effects.

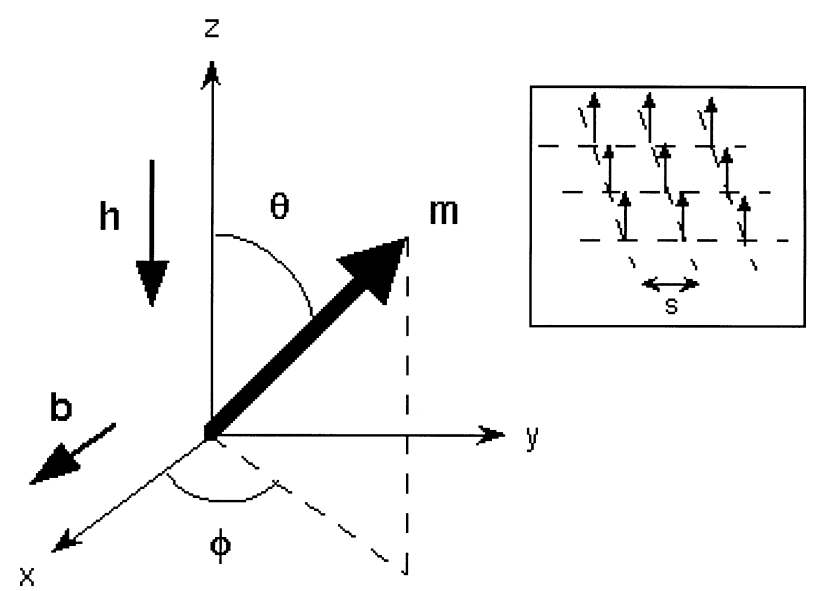

Fig.1: Geometry. The angles $\theta$ and $\phi$ specify the time dependent position of the total magnetic moment of a single domain, small magnetic particle $\mathbf{m}$. A switching field $\mathrm{h}$ is applied along the uniaxial anisotropy easy axis, and a small bias field $\mathrm{b}$ is directed along the $\mathrm{x}$ axis at time $=0$. The inset shows the geometry of a square planar array of identical particles with array spacing s.
The total magnetic moment of a particle is represented by the vector $\mathbf{m}$, and orientation angles $\theta$ and $\phi$ are defined in Fig. 1. A uniaxial anisotropy axis is taken along the $\mathrm{z}$ direction, and a switching field $\mathrm{h}$ is applied in the negative $\mathrm{z}$ direction. A small bias field $\mathrm{b}$ is also applied along the $\mathrm{x}$ direction. The figure inset contains a sketch of the particle in an array geometry, where single domain particles represented by arrows are set in a square lattice with equilibrium orientations normal to the plane.

Switching behavior of an individual magnetic moment $\mathbf{m}$ is considered. Effects of weak coupling on arrays of particles are discussed elsewhere. ${ }^{3}$ The Gilbert form of the equations of motion are used to describe the dynamics of $\mathbf{m}$ :

$$
\frac{d \vec{m}}{d t}=\gamma \vec{m} \times \vec{H}-\alpha \vec{m} \times \frac{d \vec{m}}{d t}
$$

where $\gamma$ is the gyromagnetic ratio and $\alpha$ determines the damping rate. The field $\mathbf{H}$ contains $\mathrm{h}, \mathrm{b}$, and the effective anisotropy field, defined by $\mathrm{k} \mathrm{m}_{\mathrm{Z}}=\left(2 \mathrm{~K} / \mathrm{M}^{2}\right) \mathrm{m}_{\mathrm{Z}}$ where $\mathrm{K}$ is the anisotropy energy and $\mathrm{M}$ is the magnetization. With these definitions, the field $\mathbf{H}$ is given as

$$
\mathbf{H}=\left(\mathrm{k} \mathrm{m}_{\mathrm{Z}}-\mathrm{h}\right) \mathbf{z}+\mathrm{b} \mathbf{x}
$$

The initial conditions are taken with $\mathbf{m}$ at along the $\mathrm{z}$ axis. At $\mathrm{t}=0, \mathrm{~h}$ is applied in the negative $\mathrm{z}$ direction. This is an unstable equilibrium, and an infintesimally small perturbation is sufficient to begin precession of the moment. If $\mathrm{h}$ and $\mathrm{b}$ are large enough compared to $\mathrm{K}$, the moment will eventually equilibriate along the $-\mathrm{z}$ direction.

It is well known that precession effects are important in determining thermal activated switching rates. ${ }^{4,5}$ It will be seen here that precession effects are also very important for determining driven switching rates, and precession can in fact be used to advantage in optimising the reversal rate.

It is first useful to illustrate the relationships between switching time, $\mathrm{h}, \mathrm{k}$ and $\mathrm{b}$. The precession information can be removed from the equations of motion by separating the slow time behavior of $\mathbf{m}$ from the fast time behavior with the assumption of time dependences of the form

$$
\begin{aligned}
& \mathrm{m}_{\mathrm{X}}=M \sin \theta(\mathrm{t}) \mathrm{f}(\mathrm{t}) \\
& \mathrm{m}_{\mathrm{y}}=\mathrm{M} \sin \theta(\mathrm{t}) \mathrm{g}(\mathrm{t})
\end{aligned}
$$




$$
\mathrm{m}_{\mathrm{Z}}=\mathrm{M} \cos \theta(\mathrm{t})
$$

The time dependence of $f(t)$ and $g(t)$ are the assumed to be oscillatory with periods much shorter than that of $\theta(\mathrm{t})$.

These solutions are substituted into the equations of motion (1) and terms to order one in $\alpha$ kept. The equations are next averaged over a time large compared to the period of $f(t)$ and $g(t)$ but short compared to the period of $\theta(t)$. This procedure results in a single equation describing the time evolution of $\mathbf{m}$ :

$$
\frac{1}{\alpha \gamma} \frac{d \theta}{d t}=-b \cos \theta-h \sin \theta+k \sin \theta \cos \theta
$$

In the case $b=0$ and $k=0$, the solution to this equation satisfies $\frac{d \theta}{d t}=-\alpha \gamma h \sin \theta$ and is given by

$$
\cos \theta(\mathrm{t})=-\tanh (\Delta \mathrm{t})
$$

where $\Delta=\alpha \gamma \mathrm{h}$ is a measure of the inverse switching time. Note that $\gamma \mathrm{h}$ is the precession frequency of $\mathbf{m}$ responding to torques produced by $\mathrm{h}$. The effect of a bias field $\mathrm{b}$ with $\mathrm{k}=0$ is to decrease the switching time by increasing the torque on $\mathbf{m}$ for small $\theta$. The increase in torque is directly proportional to $\mathrm{b}$ at small $\theta$, and one can show, by solving Eq. (6) and using $\mathrm{b} / \mathrm{h} \ll 1$, that the switching time $\tau_{\mathrm{S}}$ behaves as 6

$$
\tau_{\mathrm{s}}=(1-\mathrm{b} / \mathrm{h}) / \Delta
$$

The switching time in Eq. (8) is defined as twice the time needed to go from $\theta=0$ to $\theta=\partial / 2$. The increase in torque due to $b$ appears as an increased precession frequency, and hence a shorter switching time.

The effect of anisotropy is to impede the switching process for $\mathrm{h}<\mathrm{k}$. One of the main points of this paper, however, is to show how this can be overcome using a small bias field. As will be explained below, switching can occur for $\mathrm{b}$ greater than some critical value even if $\mathrm{h}<\mathrm{k}$ and the $\theta=0$ orientation

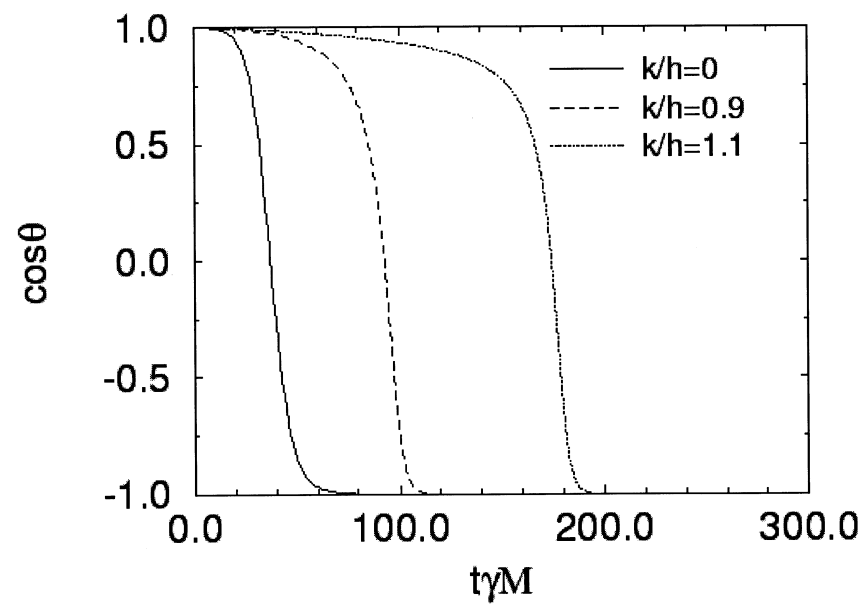

Fig.2: The component of $\mathbf{m}$ along the switching field as a function of time for different values of the anisotropy. These results were obtained in the limit of large damping using the approximation described in the text. The bias field has magnitude $\mathrm{b} / \mathrm{h}=0.05$. Alignment with the switching field will only occur for $\mathrm{b}$ above a critical value if $\mathrm{k}>\mathrm{h}$. is stable.

It is difficult to find a closed form solution to Eq. (6) with non-zero anisotropy, but a numerical solution can be readily made. Results are shown in Fig. 2 where the time dependence of $\mathrm{m}_{\mathrm{Z}} / \mathrm{M}=\cos \theta$ is found by numerically integrating Eq. (6) using different values of $\mathrm{k} / \mathrm{h}$. The other parameters are $\mathrm{h} / \mathrm{M}=0.8, \mathrm{~b} / \mathrm{h}=0.05$ and $\gamma / \alpha=10$. Time is given in reduced units $\mathrm{t} \gamma \mathrm{M}$.

The case $\mathrm{k}=0$ is shown as a reference, and displays a profile for $\mathrm{m}_{\mathrm{Z}}$ that reflects the asymmetry about the $\theta=ð / 2$ point. The effect of anisotropy is to distort the profile and slow the initial switching process, thereby increasing the entire switching time. It is also interesting to note that when $b=0$ switching will eventually occur for $\mathrm{k} / \mathrm{h}>1$, but will not occur for $\mathrm{k} / \mathrm{h}>1$. The reason is that a local minimum in the energy at $\theta=0$ exists when $\mathrm{k}>\mathrm{h}$. The transverse field $\mathrm{b}$ distorts the local minimum and lowers the energy barrier for positive $\theta$. When $b$ is large enough, the barrier is negated and the minimum at $\theta=0$ becomes unstable, thereby allowing switching. In this example, $b<0.02$ will not lead to switching for the $\mathrm{k} / \mathrm{h}=1.1$ curve shown in Fig. 2 .

The analysis so far has assumed that switching occurs over time scales large compared to the period of precession. The dynamics of precession can actually decrease the switching time and result in critical damping, at least when $\mathrm{k}<\mathrm{h}$, as would be expected for a damped oscillator. Critical damping does not exist for $\mathrm{k}>\mathrm{h}$, as will be shown below, but precession can substantially aid reversal instigated with a bias field.

Numerical simulations were made by integrating the full Landau Lifschitz equations of motion for a precessing $\mathbf{m}$ in order to examine the critically damped region. The method is straightforward and details are given in Ref. 7. The numerical work allows one to qualify the severity of the approximations made in the above slow time approximation. Results from the two different methods were found to agree in the limit $\gamma / \alpha<<0$.

The effects of precession are shown in Fig. 3 where $m_{x}, m_{y}$ and $\mathrm{m}_{\mathrm{Z}}$ are shown as functions of time for $\mathrm{k} / \mathrm{h}=1.1$, $\mathrm{b} / \mathrm{h}=0.05$ and $\gamma / \alpha=50$ as calculated numerically from the Landau Lifshitz equations. The parameters are the same as

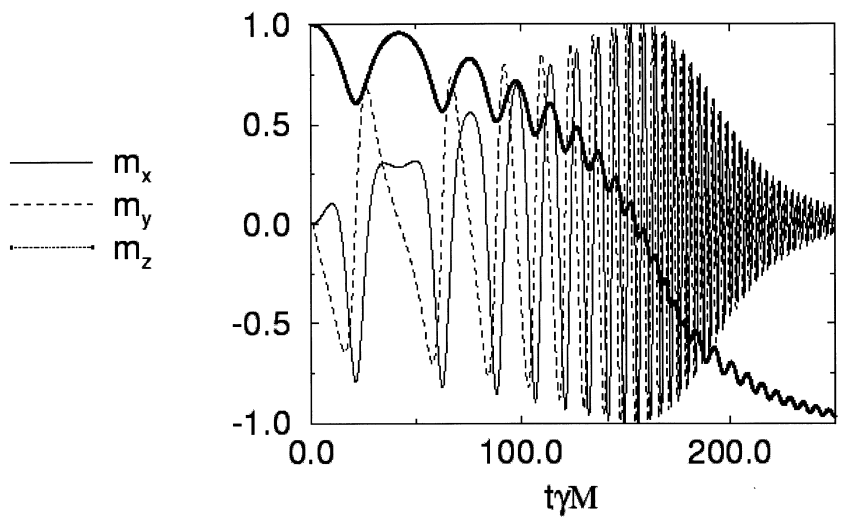

Fig.3: Effect of precession on reversal. The dark solid line is $\mathrm{m}_{\mathrm{Z}} / \mathrm{M}$, the light solid line is $\mathrm{m}_{\mathrm{X}} / \mathrm{M}$ and the dashed line is $\mathrm{m}_{\mathrm{y}} / \mathrm{M}$. The parameters are: $\mathrm{k} / \mathrm{h}=1.1$, $\mathrm{b} / \mathrm{h}=0.05$, and $\gamma / \alpha=50$. These results were obtained by numerically integrating the full equations of motion. Precession of $\mathbf{m}$ decreases the switching time and appears as high frequency oscillations in the components of $\mathbf{m}$. 


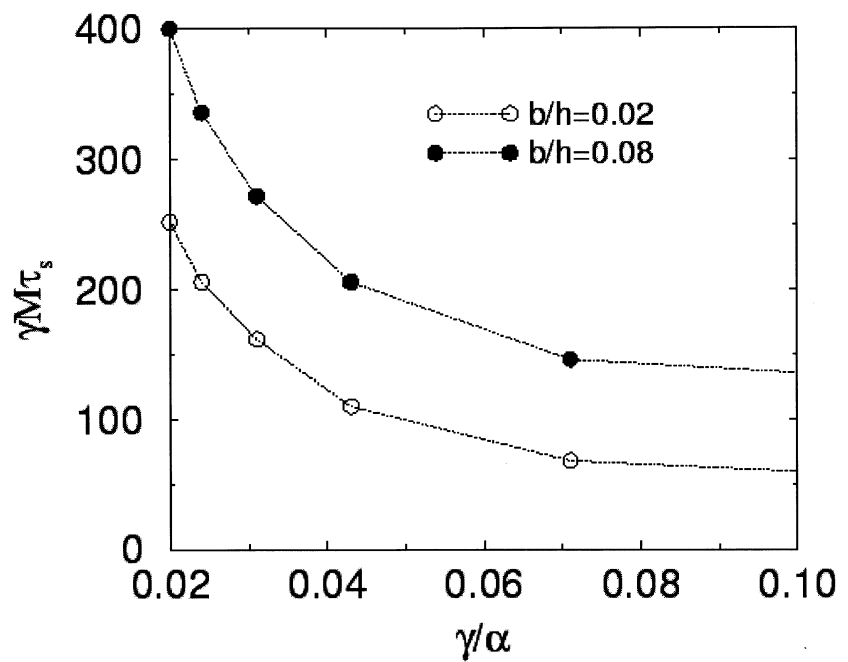

Fig.4: Switching time versus $\gamma / \alpha$ for $\mathrm{k} / \mathrm{h}=1.1$. The switching time $\tau_{\mathrm{S}}$ (defined as twice the time needed to go from $\theta=0$ to $\theta=ð / 2$ ) increases rapidly as $\gamma$ is reduced. There is no critical damping since $\mathrm{k}>\mathrm{h}$, and $\tau_{\mathrm{S}}$ approaches limiting

values as $\alpha$ increases.

those used in Fig. 2 except for the $\gamma / \alpha$ ratio. Note that the increase in switching time due to the increase in $\gamma / \alpha$ is largely countered by the effects of precession. Thus even though damping is decreased by a factor of five, the switching time increases only slightly. By way of contrast, results using the previous ratio of $\gamma / \alpha=10$ give a switching time of about 50, compared to the result of nearly 200 obtained using the fast time average approximation of Eq. (6).

The dependence of switching time on $\gamma / \alpha$ is shown in Fig. 4 for two different bias fields $b / h$. The results are calculated using the numerical simulation as in Fig. 3. The anisotropy is again $\mathrm{k} / \mathrm{h}=1.1$, so that switching will not occur unless $b$ is greater than some minimum value. In this case there is no critical damping since $\mathrm{k}>\mathrm{h}$, and instead the switching times approach limiting values as $\gamma / \alpha$ increases.
Note that switching time decreases slowly for $\gamma / \alpha$ ratios greater than 0.08 .

It is also useful to note that the same results can be obtained using short pulses for $b$. The length of the pulse need only be enough to force $\mathbf{m}$ out of the anisotropy induced energy well at $\theta=0$. The length is approximately the time needed to go from $\theta=0$ to $\theta=ð / 2$, or one half $\tau_{\mathrm{s}}$.

All of the previous discussion considered only the switching of single, isolated magnetic particles. Because of stray dipolar fields, collections of magnetic particles can interact over fairly large distances. This can complicate the dynamics of particle precession and strongly affect switching times. ${ }^{4}$

In conclusion, an optimized switching time for the magnetization of single domain particles has been found when a small perturbing bias field is applied. If the bias is sufficiently large, it can destabilize the magnetization and lead to reversal even when the switching field is less than the anisotropy field. Effects of precession and damping on the reversal process were examined and relations between switching time and bias field were derived in special cases.

\section{Acknowledgements:}

RLS acknowledges support under ARC Small Grant, NSF INT-9603252 and DMR-9703783. BH acknowleges support under the Deutsche Forschungsgemeinschaft.

\section{References}

[1] M. Hehn, K. Ounadjela, J.P. Bucher, F. Rousseaux, D. Decanini, B. Bartenlian, C. Chappert, Science 272, 1782 (1996).

[2] W. K. Hiebert, A. Stankiewicz, M. R. Freeman, Phys. Rev. Lett. 79, 1134 (1997).

[3] R. L. Stamps, R. E. Camley, unpublished.

[4] W. F. Brown, Jr., Phys. Rev. 130, 1677 (1963).

[5] R. Kikuchi, J. Appl. Phys. 27, 1352 (1956).

[6] The solution to Eq. (6) can be easily found by integration, and is given by $\tan (\theta / 2)=\left[h-\left(b^{2}+h^{2}\right) 1 / 2\right] \tanh \left[\alpha\left(b^{2}+h^{2}\right) 1 / 2 t / 2\right] / b$.

[7] M. Bauer, B. Hillebrands, R. L. Stamps, unpublished. 